\section{Bewährte und neue Methoden passend kombinieren}

\section{"Automation von ALKIS und ATKIS ist in hohem Grad möglich"}

Ein Interview mit Prof. Dr. M. Sester durchgeführt von: Waldemar Meissner, Robert Lörks, Sabine Parschau

Das Thema Automation in der Kartographie war ein Schwerpunkt des Landmanagement Forums, dem diesjährigen Kundentag der AED-SICAD AG. In einem Gastvortrag beschäftigte sich Professor Monika Sester vom Institut für Kartographie und Geoinformatik der Leibniz Universität Hannover mit der Automation in der Kartographie und weiteren interessanten Themen aus der Forschung. In einem Interview haben wir einige Aspekte des Vortrags nochmals aufgegriffen.

gis@work (g@w): Womit befassen Sie sich in Ihren aktuellen Forschungsarbeiten?

Sester: Einer unserer Schwerpunkte ist die Interpretation von Bewegungstrajektorien. Wir fragen uns, wie wir aus Spuren, die Menschen, Tiere oder Fahrzeuge hinterlassen haben, sinnvolle Informationen ableiten können. Ein weiteres großes Gebiet ist das Mobile Mapping und die daraus ableitbaren Daten und Anwendungen, insbesondere im Bereich Automotive. Das geht stark in Richtung autonomes Fahren. Unter kartographischen Aspekten beschäftigen wir uns mit neuen Methoden u. a. zur Abbildung der Dynamik, aber auch der Erweiterung schon vorhandener Methoden zur Datenintegration. Hier müssen wir uns ständig neuen Herausforderungen stellen und moderne Konzepte entwickeln.

g@w: Im Bereich der Generalisierung sind viele Verfahren an Ihrem Institut entwickelt worden, ein Teil davon ist auch in unsere 3A-Produkte integriert. Mit Fokus auf ALKIS und ATKIS: Wo sehen Sie die Zukunft dieser beiden Modelle?

Sester: Wichtig ist, dass man die Zusammenhänge zwischen den Modellen herstellt. Jedes Modell hat seine Bedeutung und seine Anwendungen. Aber aus vielerlei, auch wirtschaftlicher Hinsicht ist es wichtig, Synergien zu finden und diese Daten nicht getrennt zu halten und fortzuführen. Es gibt Ansätze, die diesen Konflikt lösen könnten, aber an manchen Stellen bleibt es schwierig. Wenn man beide Datenbestände als solche haben möchte, wird man an manchen Stellen in Kauf nehmen müssen, Zusatzerfassungen zu haben. Aber vieles lässt sich automatisieren.

g@w: Es gibt die Vision eines einheitlichen Geodatenbasis bestandes bis 2030 (GeoBasisDE 2030). Wie könnte ein solcher einheitlicher Datenbestand geschaffen werden?

Sester: Man kann die Unterschiede zwischen den Modellen, wenn sie regelbasiert sind, sehr gut in Software abbilden und implementieren. Hier sehe ich keine Schwierigkeiten. Wenn die Regeln nicht bekannt sind, können Lernverfahren eingesetzt werden. Das scheint aus unserer Sicht ein sehr vielversprechender Ansatz.

g@w: Dann gehen Sie davon aus, dass man die Verfahren grundsätzlich verbinden kann? Beim maschinellen Lernen sind ja keine Regeln direkt ableitbar?

Sester: Man muss wissen, wann welche Methode einzusetzen ist. Nur dort, wo ich mir zu 100 Prozent sicher bin, dass ich die Regeln kenne, würde ich diese explizit als Regelwerk einführen. Dort, wo es Grenzfälle gibt, würde ich die maschinellen Lernverfahren anwenden. Aus diesen Verfahren versprechen wir uns nicht nur eine Klassifikation, sondern erwarten auch homogenere Entscheidungen und damit eine höhere Sicherheit.

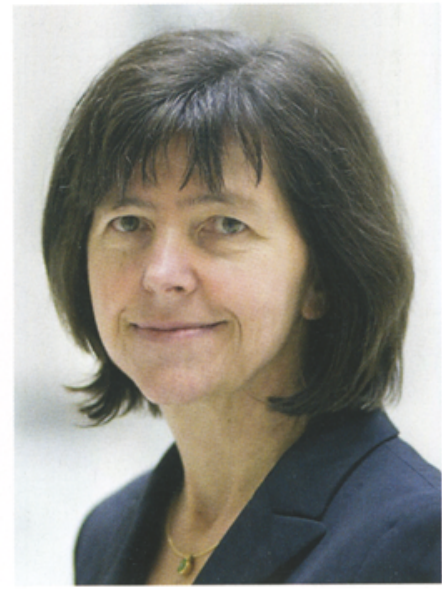

Prof. Monika Sester

g@w: Gibt es für die Methode Deep Learning für Generalisierungszwecke schon konkrete For schungsbeispiele?

Sester: Speziell in der geometrischen Generalisierung noch nicht. Aber im Bereich der Klassifikation gibt es sehr viele Ansätze.

g@w: Verstehen Sie die Methoden, die Sie in Ihrem Haus zur geometrischen Homogenisierung und automatischen Generalisierung von ALKIS und ATKIS anwenden, als vorbereitende Maß- nahme zur GeoBasisDE 2030? Sind das die ersten Schritte zu einem einheitlichen Datenbestand?

Sester: Es ist sicher die Intention der Landesvermessung, zunächst einmal möglichst viele Daten konsistent überlagern zu können. Dann kann man die Diskrepanzen deutlicher erkennen und überlegen, wie hiermit weiter verfahren wird.

g@w: Welcher Automatisierungsgrad ist Ihrer Ansicht nach erreichbar?

Sester: Grundsätzlich glaube ich schon, dass man zu einem hohen Grad kommen kann. Die wesentlichen Operatoren für eine Homogenisierung sind grundsätzlich verfügbar. Es bleibt aber die grundlegende Frage nach der Zielvorstellung: Möchte ich Generalisierungsregeln, die mir dann ein anderes ATKIS-Modell liefern, das aber auch konform ist? Je genauer ich hier Zielvorgaben bzw. Rahmenbedingungen für die Prozesse mache, umso höher wird der Grad der Automatisierung.

\section{Smart Production}

\section{Karten stets aktuell und in bester Qualität}

Ein Praxisbericht über die automatische und integrierte Herstellung sowie Aktualisierung von deutschen topographischen Kartenprodukten

Ajay Mathur, Siegen

Trotz Standardisierung des Datenmodells und der Signaturenkataloge sind Unterschiede in der technischen Umsetzung der Produktionsprozesse bei den deutschen Bundesländern festzustellen. Dies wiederum führt zu Unterschieden bei der Qualität und Aktualität der Topographischen Kartenprodukte und zu mangelnden Homogenitäten. Dabei verwendet die Mehrheit der Bundesländer teilweise unterschiedliche Technologien für die automatische Generalisierung um Kartendaten aus ihren AAA-Geobasisdaten zu erzeugen. Eine hochwertige automatische Generalisierung kann Ergebnisse in hoher Qualität liefern und dadurch deutliche Zeitersparnisse bei der Karten-Erstherstellung und der darauffolgenden händischen Bearbeitung mit sich bringen. Damit wird die Herstellungseffizienz erhöht. Aber wie sieht es mit der Aktualität der Kartenprodukte aus?

Es ist unbestritten, dass der Nutzen einer automatischen Generalisierung nachhaltiger und dauerhaft größer wird, wenn die automatische Generalisierung als Grundlage für eine regelmäßige und beliebig wiederkehrende automatische Aktualisierung der Karten- 